\title{
Função urinária e sexual de mulheres puerperais que usaram o balão vaginal na preparação para o parto vaginal - um estudo transversal
}

\author{
Urinary and sexual function of puerperal women who \\ used the vaginal balloon in preparation for vaginal \\ birth - a cross sectional study
}

\author{
Camila Alcântara Fernandes ${ }^{1}$ \\ Isabela Lima Cavalcante ${ }^{2}$ (1) \\ Ana Clara Nunes Soares ${ }^{3}$ (1) \\ Carla Cristina Alvarez Serrão 4 (1)
}

\section{Pedro Sávio Macêdo de Almeida 5 \\ Nazete dos Santos Araújo ${ }^{6}$ (c) Gustavo Fernando Sutter Latorre 7 (1) Erica Feio Carneiro Nunes ${ }^{8}$ (1)}

1-4Universidade do Estado do Pará (Belém).Pará, Brasil. camisfernandes10@gmail.com, isabelacavalcante04@gmail.com, ana.claranunes@hotmail.com, cserrao18@yahoo.com.br

${ }^{5}$ Centro Universitário do Pará (Belém). Pará, Brasil. pedrosaviofisio@gmail.com 6Universidade da Amazônia (Belém). Pará, Brasil. nazetearaujo@hotmail.com

${ }^{7}$ Portal Perineo.net (Florianópolis). Santa Catarina, Brasil. gustavo@perineo.net ${ }^{8}$ Autora para correspondência. Universidade do Estado do Pará (Belém). Pará, Brasil. erica@perineo.net

RESUMO | INTRODUÇÃo: O balão vaginal pode ser uma opção para preparar os músculos do assoalho pélvico para o parto vaginal. Suas implicações para as funções urinária e sexual precisam ser mais estudadas. OBJETIVO: Avaliar as implicações do balão vaginal na preparação para o parto vaginal nas funções sexual e urinária. METODOLOGIA: Estudo transversal realizado em Belém do Pará, com primíparas de partos vaginais, de 18 a 40 anos. Formouse dois grupos: o GE se constituiu de puérperas que usaram o balão vaginal durante a gravidez provenientes da Clínica Cafisio Mulher e o GC de puérperas que não fizeram uso do balão vaginal durante a gestação, provenientes do Centro de Saúde Escola do Marco. Utilizou-se uma ficha de avaliação sociodemográfica, um questionário com perguntas acerca dos eventos do trabalho de parto, o International Consultation on Incontinence Questionnaire Short Form e o Female Sexual Function Index. Foi utilizado o software Excel para entrada dos dados, confecção das tabelas e análise estatística descritiva das variáveis. RESULTADOS: Em ambos os grupos, a maioria das mulheres apresentou alguma perda urinária, com impactos diferentes na qualidade de vida. Quanto à função sexual, ambos os grupos estão abaixo do ponto de corte, o que indica disfunção sexual. No entanto, não foi possível estabelecer uma associação entre a função urinária e sexual entre os dois grupos devido às diferenças no tamanho da amostra entre os grupos. CONCLUSÃo: As funções urinária e sexual de ambos os grupos estavam alteradas.

PALAVRAS-CHAVE: Assoalho pélvico. Mulheres grávidas. Parto.
ABSTRACT | INTRODUCTION: Vaginal balloon can be an option to prepare the pelvic floor muscles for vaginal birth. Its implications for urinary and sexual functions need to be further studied. OBJECTIVE: To evaluate the implications of the vaginal balloon in preparation for vaginal birth on sexual and urinary functions. METHODOLOGY: Cross-sectional study was carried out in Belém do Pará, with primiparous women from vaginal births, aged between 18 and 40 years. Two groups were formed: the EG consisted of postpartum women who used the vaginal balloon during pregnancy from the Clínica Cafisio Mulher and the CG of postpartum women who did not use the vaginal balloon during pregnancy, from the Centro de Saúde Escola do Marco. A sociodemographic evaluation form, a questionnaire with questions about labor events, the International Consultation on Incontinence Questionnaire Short Form, and the Female Sexual Function Index were used. Excel software was used for data entry, preparation of tables, and descriptive statistical analysis of the variables. RESULTS: Most women had some urinary loss in both groups, with different impacts on quality of life. As for sexual function, both groups are below the cutoff point, which indicates sexual dysfunction. However, it was impossible to establish an association between urinary and sexual function between the two groups due to differences in sample size. CONCLUSION: The urinary and sexual functions of both groups were altered.

KEYWORDS: Pelvic floor. Pregnant women. Childbirth. 


\section{Introdução}

O assoalho pélvico (AP) é composto por músculos, ligamentos e fáscias, com o importante papel de suspensão e sustentação dos órgãos pélvicos, bem como de auxiliar na função sexual e manter a continência fecal e urinária. ${ }^{1}$

Todavia, determinadas situações tendem a predispor esse conjunto de estruturas a modificações anatomofisiológicas, a exemplo do que ocorre no ciclo

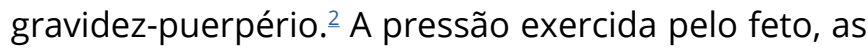
efetivas contrações uterinas durante a descida da cabeça fetal e as forças geradas pelo esforço expulsivo materno podem desencadear estiramentos da estrutura pélvica e inclusive induzir a rupturas parciais ou totais dos componentes do AP, causando variados tipos de lesões e prejuízos funcionais. ${ }^{3}$

Sendo assim, a fim de evitar quadros lesivos na região e manter ao máximo sua funcionalidade ligamentar e muscular, entende-se que os músculos do assoalho pélvico (MAP) precisam estar tônicos para sustentar o bebê durante a gestação, contudo devem também estar elásticos, para que possam se distender adequadamente no momento do parto. ${ }^{4}$ Para a tonicidade há a adoção do protocolo de Kegel, cujo intuito é exercitar a musculatura perineal e trabalhar sua hipotonia; enquanto que para a elasticidade há outras estratégias, a exemplo dos balões vaginais, como o Epi-No.,

O Epi-No é um dilatador vaginal formado por um balão de silicone inflável que se conecta a um manômetro de pressão e a uma bomba manual, de modo que quando pressionada oferta ar comprimido, gerando assim o crescimento ou expansão desse balão. Acredita-se que essa expansão, dentro do limiar de tolerância de cada mulher, acarrete em alongamento das estruturas perivaginais e do períneo, o que por sua vez favoreceria a passagem do feto por uma maior distensão do canal de parto. Ainda apresenta a finalidade de treinar a mãe para o período expulsivo, podendo diminuir o tempo desse estágio do trabalho de parto e, consequentemente a necessidade da utilização de analgésicos para alívio de dor. $\underline{7}$
Já foi observada a efetividade desse instrumento na extensibilidade do canal vaginal no período gestacional6, sem alterar a resistência dos músculos do assoalho pélvico (MAP). Entretanto, há poucos dados e pesquisas referentes às suas implicações na função urinária e sexual, de modo que uma explanação sobre esses aspectos seria enriquecedora, tanto para o âmbito acadêmico e profissional quanto para as próprias puérperas, uma vez que aumentaria o arsenal de recursos disponíveis para uma preparação de qualidade para o parto.

Diante do exposto, o presente estudo teve por objetivo avaliar as implicações do balão vaginal nas funções sexual e urinária de puérperas que o utilizaram como preparação para o parto.

\section{Material e métodos}

Trata-se de um estudo observacional descritivo do tipo transversal, aprovado pelo Comitê de Ética em Pesquisa com Seres Humanos do Centro de Ciências Biológicas e da Saúde da Universidade do Estado do Pará (CAAE: 09087419.8.0000.5174, Parecer $n^{\circ}$ 3.333.014), ocorrido na clínica de Fisioterapia CAFISIO Mulher e no Centro de Saúde Escola do Marco (CSEMarco), ambos em Belém do Pará.

A coleta de dados ocorreu de setembro de 2019 a outubro de 2020, tendo como público alvo puérperas primíparas de parto vaginal que fizeram ou não o uso do balão vaginal durante a gestação. A amostragem foi por conveniência, de forma que para o GE foi consultada uma lista de mulheres da CAFISIO-Mulher que fizeram a preparação para parto vaginal com o balão vaginal e foram selecionadas as que se encaixavam nos critérios de inclusão da pesquisa. Após, houve o contato telefônico para convidá-las a participarem e quem aceitasse deveria se dirigir à clínica para a avaliação. Para o GC, as puérperas eram abordadas na fila para consulta no CSE-Marco, e as que aceitavam eram encaminhadas para uma sala reservada para aplicação dos questionários. 
Foram formados dois grupos: o GE (Grupo balão vaginal) e o GC (Grupo Controle), sendo que o GE se constituiu de puérperas que usaram o balão vaginal durante a gravidez provenientes da Clínica Cafisio Mulher e o GC se constituiu de puérperas primíparas que não fizeram uso do balão vaginal durante a gestação, provenientes do CSE-Marco.

Foram incluídas em ambos os grupos puérperas primíparas de 18 a 40 anos, de modo que no GE estavam aquelas que fizeram uso do balão vaginal durante a gestação para o parto normal e no GC as que não usaram. Foram excluídas em ambos os grupos puérperas que tiveram gravidez de risco, gestação múltipla, sejam multíparas e/ ou que realizaram qualquer outro tipo de parto.

Ambos os grupos responderam a uma ficha de avaliação sociodemográfica criada pelos pesquisadores; um questionário com perguntas acerca dos eventos do trabalho de parto. Para a avaliação da função urinária foi usado o International Consultation on Incontinence Questionnaire Short Form (ICIQ -SF), que se trata de um questionário simples, breve e autoadministrável constituído por duas questões de identificação, 3 questões sobre a quantidade e frequência da perda de urina, e uma que questiona em quais situações ocorre essa perda, de modo que quanto maior o escore, maior é a afecção da função urinária. . $^{-}$

Por fim, para a investigação da função sexual das puérperas, foi adotado o Female Sexual Function Index (FSFI), responsável por avaliar o desejo sexual, excitação, lubrificação vaginal, orgasmo, satisfação e dor. De modo que quando somados os escores de cada domínio, são multiplicados por um fator que homogeneíza a influência de cada domínio, e, ao final, chega-se ao escore total (Quadro 1), no qual quanto maior o valor, melhor a função, sendo considerado o escore de corte de 26,55 para a presença ou não de disfunção sexual..

Quadro 1. Escores de acordo com o domínio do FSFI

\begin{tabular}{|c|c|c|c|c|c|}
\hline Domínio & Questão & Variação do escore & Fator & Escore Mínimo & Escore Máximo \\
\hline Desejo & 1,2 & $1-5$ & 0,6 & 1,2 & 6,0 \\
\hline Excitação & $3,4,5,6$ & $0-5$ & 0,3 & 0 & 6,0 \\
\hline Lubrificação & $7,8,9,10$ & $0-5$ & 0,3 & 0 & 6,0 \\
\hline Orgasmo & $11,12,13$ & $0-5$ & 0,4 & 0 & 6,0 \\
\hline Satisfação & $14,15,16$ & 0 (ou 1$)-5^{\star}$ & 0,4 & 0,8 & 6,0 \\
\hline Dor & $17,18,19$ & $0-5$ & 0,4 & 0 & 6,0 \\
\hline Escore Total & & & 2,0 & 36 \\
\hline
\end{tabular}

*Variação para o item 14 = 0-5; variação para os itens 15 e 16 = 1-5.

O balão vaginal utilizado no período gestacional foi o Epi-No, da seguinte forma: início do uso com 32 semanas até o parto, uma vez por semana, de 5 a 10 minutos, em consultório com a fisioterapeuta. Gradualmente se inflava o balão em média de 0,5 a $1 \mathrm{~cm}$ de diâmetro a cada sessão. As gestantes treinavam com o balão em decúbito dorsal até a $35^{\mathrm{a}}$ semana e em bipedestação até o dia do parto (protocolo da Cafisio Mulher).

Foi utilizado o software Excel para entrada dos dados, confecção das tabelas e análise estatística descritiva das variáveis, que foram apresentadas por meio de frequência relativa e medidas de tendência central (média).

\section{Resultados}

Participaram do estudo 15 puérperas, sendo 4 no grupo GE e 11 no grupo GC. A tabela 1 apresenta o perfil sociodemográfico da amostra, evidenciando uma média de idade $\mathrm{GE}=36,5 \pm 5,0$ anos, e $G C=28+6.7$ anos. Em ambos os grupos a maioria era casada, possuía algum tipo de ocupação e oito ou mais anos de estudo (Tabela 1). 
Tabela 1. Perfil sociodemográfico da amostra

\begin{tabular}{|c|c|c|}
\hline \multirow{2}{*}{ Variáveis } & \multicolumn{2}{|c|}{ Frequência relativa } \\
\hline & GE $(n=4)$ & GC $(n=11)$ \\
\hline Idade (Média e DP) anos & $36,5 \pm 5,0$ & $28 \pm 6.7$ \\
\hline \multicolumn{3}{|l|}{ Estado civil } \\
\hline Solteira & - & $20,0 \%$ \\
\hline Casada & $100,0 \%$ & $60,0 \%$ \\
\hline Divorciada & - & $20,0 \%$ \\
\hline \multicolumn{3}{|l|}{ Ocupação } \\
\hline Administradora & - & $18,2 \%$ \\
\hline Assistente administrativa & - & $9,1 \%$ \\
\hline Cirurgiã dentista & - & $9,1 \%$ \\
\hline Dona de casa & - & $9,1 \%$ \\
\hline Estudante & - & $27,3 \%$ \\
\hline Recepcionista & - & $9,1 \%$ \\
\hline Universitária & - & $9,1 \%$ \\
\hline Vendedora & $14,3 \%$ & $9,1 \%$ \\
\hline Empresária & $28,6 \%$ & $25,0 \%$ \\
\hline Médica & $14,3 \%$ & $25,0 \%$ \\
\hline Professora & - & $25,0 \%$ \\
\hline Psicóloga & - & $25,0 \%$ \\
\hline \multicolumn{3}{|l|}{ Religião } \\
\hline Católica & $72,7 \%$ & $27,3 \%$ \\
\hline Evangélica & $9,1 \%$ & $36,4 \%$ \\
\hline Outros & $18,2 \%$ & $36,4 \%$ \\
\hline \multicolumn{3}{|l|}{ Cor } \\
\hline Branca & $12,5 \%$ & $8,3 \%$ \\
\hline Parda & $50,0 \%$ & $50,0 \%$ \\
\hline Amarela & $37,5 \%$ & $25,0 \%$ \\
\hline Negra & - & $16,7 \%$ \\
\hline \multicolumn{3}{|l|}{ Escolaridade } \\
\hline Médio completo & - & $56,0 \%$ \\
\hline Superior incompleto & - & $20,0 \%$ \\
\hline Superior completo & $46,2 \%$ & $24,0 \%$ \\
\hline Pós graduação & $53,8 \%$ & - \\
\hline
\end{tabular}

(-) Dados numéricos igual a zero.

Na história obstétrica das participantes, observou-se que a maioria não sofreu nenhum aborto, tendo tipo apenas uma gestação. Todas elas realizaram o pré-natal, com média de 9,06 consultas. Porém, observou-se uma grande disparidade entre os dois grupos quanto à preparação com fisioterapeuta, pois enquanto $100 \%$ do grupo GE realizou algum tipo de preparo fisioterapêutico, com o GC ocorreu o oposto (Tabela 2).

Tabela 2. História obstétrica da amostra.

\begin{tabular}{|c|c|c|c|}
\hline \multirow{2}{*}{\multicolumn{2}{|c|}{ Variáveis }} & \multicolumn{2}{|c|}{ Frequência relativa } \\
\hline & & $G E(n=4)$ & $G C(n=11)$ \\
\hline \multirow{2}{*}{ Aborto } & Sim & $33,3 \%$ & $9,1 \%$ \\
\hline & Não & $66,7 \%$ & $90,9 \%$ \\
\hline \multirow{2}{*}{ Número de gestações } & 1 & $66,7 \%$ & $90,9 \%$ \\
\hline & 2 & $33,3 \%$ & $9,1 \%$ \\
\hline \multirow{3}{*}{$\begin{array}{l}\text { Número de semanas que } \\
\text { tiveram o bebê }\end{array}$} & $36-38$ & $75,0 \%$ & $54,6 \%$ \\
\hline & $39-42$ & $25,0 \%$ & $45,5 \%$ \\
\hline & $6-10$ & $100,0 \%$ & $9,1 \%$ \\
\hline \multirow[t]{2}{*}{ Consultas pré-natal } & $11-14$ & - & $15,2 \%$ \\
\hline & $30-43$ & - & $18,2 \%$ \\
\hline Preparação com & $\operatorname{sim}$ & $100 \%$ & - \\
\hline fisioterapia & Não & - & $100 \%$ \\
\hline \multirow{2}{*}{$\begin{array}{l}\text { Se não, teve outro tipo } \\
\text { de preparação? }\end{array}$} & $\operatorname{sim}$ & - & $10,0 \%$ \\
\hline & Não & - & $90,0 \%$ \\
\hline Prepararia seu corpo se & $\operatorname{sim}$ & - & $100,0 \%$ \\
\hline $\begin{array}{l}\text { tivesse tido } \\
\text { oportunidade? }\end{array}$ & Não & & - \\
\hline
\end{tabular}

(-) Dados numéricos igual a zero. 
$\mathrm{Na}$ tabela 3, são evidenciados os eventos ocorridos durante o trabalho de parto das participantes. Em relação a realização de episiotomia, não se observou diferença entre os dois grupos, uma vez que no GE metade das muIheres sofreu algum corte durante o parto, e no GC a episiotomia esteve presente em somente um pouco mais de $50 \%$ delas, evidenciando dados muitos semelhantes entre grupos. Uma variável discrepante foi a posição adotada no trabalho de parto, pois apesar de a maioria das mulheres do GC $(72,7 \%)$ ter tido o bebê na posição convencional em decúbito dorsal com as pernas flexionadas, também houve registros de posturas verticais, como sentada/reclinada e de cócoras. Todavia, $100 \%$ do GE adotou a postura deitada.

Tabela 3. Eventos do trabalho de parto da amostra

\begin{tabular}{|c|c|c|c|}
\hline \multicolumn{2}{|c|}{ Variáveis } & \multicolumn{2}{|c|}{ Frequência relativa } \\
\hline & & GE $(n=4)$ & $G C(n=11)$ \\
\hline \multicolumn{4}{|l|}{ Semanas de gravidez } \\
\hline 36 & & $25,0 \%$ & $9,1 \%$ \\
\hline 37 & & - & $9,1 \%$ \\
\hline 38 & & $50,0 \%$ & $36,4 \%$ \\
\hline 39 & & $25,0 \%$ & $18,2 \%$ \\
\hline 40 & & - & $9,1 \%$ \\
\hline 41 & & - & $9,1 \%$ \\
\hline 42 & & - & $9,1 \%$ \\
\hline \multirow{2}{*}{ Episiotomia } & Sim & $50,0 \%$ & $54,5 \%$ \\
\hline & Não & $50,0 \%$ & $45,5 \%$ \\
\hline \multirow{3}{*}{$\begin{array}{c}\text { Posição no trabalho de } \\
\text { parto }\end{array}$} & $\begin{array}{c}\text { Deitada de costas com as } \\
\text { pernas levantadas }\end{array}$ & $100,0 \%$ & $72,7 \%$ \\
\hline & Sentada/reclinada & - & $18,2 \%$ \\
\hline & Cócoras & - & $9,1 \%$ \\
\hline
\end{tabular}

Em relação à função urinária das participantes, em ambos os grupos a maioria já vivenciou alguma perda urinária. É importante mencionar que grande parte dos registros de perda se traduzem apenas em uma pequena quantidade de urina segundo a percepção das puérperas, realidade observada de forma semelhante nos dois grupos. Porém, apesar dessa perda ser parecida entre grupos, a repercussão se mostrou bastante heterogênea, uma vez que $100 \%$ do GE afirmou que esses eventos não afetam sua vida (nenhum impacto), em contraste com os $45,5 \%$ do GC, que relatou sofrer com esse cenário, com média de 7,45, o que expressa um impacto grave (Tabela 4).

Tabela 4. Função urinária da amostra.

\begin{tabular}{|c|c|c|c|}
\hline \multicolumn{2}{|c|}{ Variáveis } & \multicolumn{2}{|c|}{ Frequência relativa } \\
\hline & & GE $(n=4)$ & $G C(n=11)$ \\
\hline \multirow{5}{*}{$\begin{array}{l}\text { Frequência da perda } \\
\text { urinária }\end{array}$} & Nunca & $25,0 \%$ & $27,3 \%$ \\
\hline & $\begin{array}{l}\text { Uma vez por semana ou } \\
\text { menos }\end{array}$ & $50,0 \%$ & $36,4 \%$ \\
\hline & $\begin{array}{l}\text { Duas ou três vezes por } \\
\text { semana }\end{array}$ & - & $9,1 \%$ \\
\hline & Uma vez ao dia & $25,0 \%$ & $18,2 \%$ \\
\hline & O tempo todo & - & $9,1 \%$ \\
\hline \multirow{3}{*}{$\begin{array}{l}\text { Quantidade da perda } \\
\text { urinária }\end{array}$} & Nenhuma & $25,0 \%$ & $27,3 \%$ \\
\hline & Uma pequena quantidade & $75,0 \%$ & $63,6 \%$ \\
\hline & Uma grande quantidade & - & $9,1 \%$ \\
\hline \multirow{4}{*}{$\begin{array}{l}\text { Em que situações ocorre } \\
\text { a perda }\end{array}$} & Nunca & $25,0 \%$ & $27,3 \%$ \\
\hline & $\begin{array}{l}\text { Perco antes de chegar ao } \\
\text { banheiro }\end{array}$ & - & $18,2 \%$ \\
\hline & $\begin{array}{l}\text { Perco quando tusso ou } \\
\text { espirro }\end{array}$ & $50,0 \%$ & $45,5 \%$ \\
\hline & $\begin{array}{l}\text { Perco quando estou } \\
\text { fazendo atividades físicas }\end{array}$ & $25,0 \%$ & - \\
\hline \multirow{2}{*}{\multicolumn{2}{|c|}{$\begin{array}{l}\text { Quanto essa perda afeta a vida - média } \\
\text { ICIQ-SF escore - média }\end{array}$}} & 0 & 7,45 \\
\hline & & 2,75 & 4,27 \\
\hline
\end{tabular}


Quanto à função sexual, a média do escore geral das mulheres que usaram o balão vaginal foi de 22,3 , enquanto o daquelas que não fizeram o uso do dispositivo foi de 24,3 , portanto, ambos os grupos se encontram abaixo do ponto de corte que é de $>26,55$. Quanto à pontuação do escore geral de cada participante, $46,6 \%$ das puérperas apresentaram pontuação abaixo do ponto de corte, sendo indicativo para disfunção sexual (Tabela 5).

Tabela 5. Média dos escore por domínio e total do questionário FSFI

\begin{tabular}{lcc}
\hline \multicolumn{1}{c}{ Domínios } & GE (n=4) & Médias de cada domínio \\
& 3,9 & GC (n=11) \\
\hline Desejo & 3,45 & 4,41 \\
Excitação & 4,27 & 4,17 \\
Lubrificação & 2,8 & 4,8 \\
Orgasmo & 2,6 & 4,47 \\
Satisfação & 4,4 & 4,94 \\
Dor & 22,3 & 4,69 \\
Média escore total & 24,3 \\
\hline
\end{tabular}

\section{Discussão}

O presente estudo objetivou verificar a influência do balão vaginal utilizado na preparação para o parto nas funções urinária e sexual de primíparas. De forma geral, não houve influência significativa do balão vaginal nos desfechos urinários e sexuais tanto de primíparas que utilizaram o balão vaginal quanto nas que não usaram.

A incontinência urinária (IU) é um fenômeno bastante presente no período gestacional com uma estimativa de aproximadamente $75 \%$, mas que pode variar em razão do tipo de estudo desenvolvido, do período investigado, bem como das características socioculturais de uma população. ${ }^{10}$ Corroborando com esse raciocínio o estudo de Santini et al. ${ }^{11}$, das 950 mulheres entrevistadas, 472 apresentaram alguma queixa de perda de urina, assumindo uma prevalência de $49,68 \%$ de IU, sendo grande parte IU do tipo mista e com maior incidência no terceiro trimestre de gestação.

Dentre os fatores de risco mais prováveis de ocorrência da IU destacam-se a multiparidade, idade materna acima de 35 anos, índice de massa corpórea pré-gestacional elevado, além das adaptações gestacionais características, como as mudanças hormonais, aumento do peso uterino e da pressão sobre o AP.12

A IU pode acometer mulheres de todas as faixas etárias, porém, sua incidência tende a aumentar com o avanço da idade, o que pode estar associado à debilidade do AP, bem como das estruturas vesicais, uma vez que o processo de envelhecimento leva a uma redução em número das fibras colágenas e diminuição dos níveis hormonais. ${ }^{13}$ Esse aumento também é relatado em outros estudos. ${ }^{11,14}$ No presente trabalho, apesar de no GE as mulheres apresentarem as maiores idades e $75 \%$ delas terem IU, no GC a puérpera mais nova apresentou episódios de perda de urina, enquanto que tiveram mulheres mais velhas que nunca vivenciaram esse quadro.

Contudo, esse quadro parece diminuir de maneira considerável no puerpério quando comparado ao período gestacional. No estudo de Valeron e Amaral15, 236 puérperas foram submetidas à avaliação fisioterapêutica tendo sido constatado que $30,61 \%$ manifestaram IU na gestação, enquanto que apenas $6,8 \%$ mantiveram as perdas urinárias no pós-parto. No entanto, em outros estudos, notou-se que a prevalência de IU no puerpério foi semeIhante àquela ocorrida na gestação. $\frac{16}{}$

Quanto à função sexual, Faisal-Cury et al. ${ }^{17}$ afirmam que o pós parto traz consigo nítidas mudanças físicas e psicológicas, e nem todas as mulheres se adaptam de forma positiva a elas. Uma prova disso é que em seu estudo foi obtido um resultado em que dois terços das mulheres, ou seja, mais da metade da amostra, experimentaram significativa redução da função sexual seis meses após o parto vaginal. 
Primíparas tendem a apresentar uma maior perturbação no orgasmo, o que pode ser justificado por um enfraquecimento dos MAP, seja ainda na gestação ou durante o trabalho de parto, levando a disfunção sexual, como a hipoestesia e anorgasmia..$^{\frac{18}{5}}$ Realidade encontrada na amostra avaliada no presente trabaIho, em especial nas mulheres que fizeram uso do balão que apresentaram essa disfunção como a segunda maior queixa, sendo a de satisfação mais citada e a dispareunia foi pouco frequente. No GC as mais prevalentes foram as disfunções de desejo e de excitação. Assim também, Holanda et al. $\frac{19}{}$, em um estudo com 200 puérperas, identificaram com maior frequência: dispareunia, seguida do vaginismo, disfunção do desejo, disfunção orgásmica e, por último, a disfunção na fase de excitação.

O parto vaginal pode implicar negativamente a função sexual no puerpério, principalmente na ocorrência de lacerações espontâneas e episiotomia..$^{20}$ Esta relação entre a presença de dispareunia, parto instrumental, lacerações espontâneas de segundo e terceiro grau, episiotomia e disfunção sexual foi observada por O'Malley et al.21 em seu trabalho de coorte com 832 primíparas.

A Fisioterapia Pélvica possui estratégias eficazes para restauração da funcionalidade do AP devido às alterações promovidas pela gestação e pós-parto. No estudo de Marques et al.22 foi comparado o treinamento dos MAP em nulíparas na $28^{\mathrm{a}}$ semana gestacional e puérperas, obtendo-se como resultado uma diminuição na urgência miccional, frequência, noctúria e IU de urgência maior no grupo composto pelas gestantes. Tendo concluído que a Fisioterapia, principalmente realizada precocemente, promove resultados positivos tanto na função muscular quanto nas funções urinária e sexual.

É importante mencionar que o alongamento da região perineal não parece prevenir a incontinência ou as disfunções de cunho sexual, entretanto, pode exercer um relevante papel na prevenção de lacerações durante o trabalho de parto. Sendo assim, os dilatadores do tipo balão vaginal, como o Epi-No, foram pensados para aumentar gradativamente o diâmetro do períneo na fase final da gravidez, com a finalidade de reduzir os traumas perineais e a episiotomia indiscriminada. $\underline{23}$

Hillebrenner et al. ${ }^{24}$ desenvolveram um estudo prospectivo composto por um grupo de 50 gestantes que realizaram o treinamento com o Epi-No a partir da $37^{\mathrm{a}}$ semana gestacional e um grupo controle de muIheres que tiveram parto vaginal no mesmo hospital e no mesmo período, e comparou as taxas de episiotomia, pontuação do Apgar, duração do trabalho de parto, necessidade de analgesia durante o parto e lacerações perineais. Como resultado, obteve-se no grupo que usou o Epi-No uma menor taxa de episiotomia ( $47 \%$ vs. $82 \%$ ) e uma menor frequência de lacerações.

De maneira semelhante, Kok et al. $\frac{25}{}$ realizaram um trabalho com 31 primíparas que utilizaram o Epi-No no pré-natal, também a partir da $37^{a}$ semana, e por um tempo de 15 minutos, comparando-as com 60 mulheres que não usaram tal dispositivo. Ao final da pesquisa, observou-se que o grupo treinado com o balão vaginal obteve uma taxa de episiotomia significativamente menor (50\% vs. $93.3 \%$ ), além de uma extensão menos severa de lacerações, o que por sua vez tem importante influência na redução de repercussões urinárias e sexuais.

Porém, a preparação para o parto dentro da fisioterapia pélvica não se limita unicamente ao treinamento com balões vaginais, pelo contrário, no geral ela parte da premissa de associar diferentes técnicas fisioterapêuticas, como o treinamento dos MAP, massagem perineal, entre outros.

Geranmayeh et al. $\frac{26}{}$ dividiram as participantes em dois grupos, sendo o primeiro composto por puérperas que fizeram preparação para o parto com massagem perineal, e o segundo funcionando como controle. O grupo de massagem apresentou $27 \%$ de mulheres que não sofreram nenhum tipo de trauma perineal, contra apenas $4 \%$ das mulheres do grupo controle que tiveram períneo íntegro, de modo que no primeiro a frequência de períneo íntegro foi significativamente maior $(p=0,004)$. 
De forma semelhante no estudo de Cardozo e Cunha ${ }^{27}$ foi apresentada diferença estatisticamente significativa quanto às lesões do assoalho pélvico em mulheres que receberam massagem perineal em comparação ao grupo controle, além de menor quadro álgico e melhor dilatação vaginal após o programa de exercícios adotado, que consistiu em: exercícios respiratórios, liberação miofascial, alongamentos, exercícios cinético-funcionais e massagem terapêutica.

Como limitação deste estudo citamos a amostra pequena, o que não permitiu análises mais seguras, bem como não houve controle de outros fatores que interferem na função sexual e urinária como idade materna, peso fetal, obesidade, doenças crônicas. Ademais, não foi possível comparar a técnica do balão vaginal usado isoladamente e associado a outras técnicas fisioterapêuticas. Sendo assim, sugere-se para os próximos estudos uma comparação entre grupos de preparação do assoalho pélvico, um de treinamento apenas utilizando o balão, outro isolando outra técnica, como a massagem perineal, e um outro combinando balão e as demais modalidades, observando a eficácia delas na função urinária e sexual das mulheres, em especial com amostras maiores.

\section{Conclusão}

A gestação e o parto se mostram como importantes fatores de risco para disfunções do assoalho pélvico, em especial quando não há preparação. A fisioterapia pélvica possui alto grau de evidência e recomendação clínica, utilizando diferentes métodos que vão desde a cinesioterapia, massagem perineal, até o uso de balões vaginais, como o Epi-No.

Esse dispositivo, no geral, tende a diminuir o segundo estágio do trabalho de parto e a presença de lacerações perineais, no entanto, no presente estudo houve a presença de repercussões negativas tanto na função urinária quanto sexual das puérperas de ambos os grupos, não havendo influência significativa do dispositivo de balão vaginal nos desfechos uroginecológicos das participantes, o que pode ser justificado pelo $\mathrm{n}$ amostral pequeno.

\section{Contribuições dos autores}

Latorre GFS foi responsável pela concepção e desenho da pesquisa. Fernandes CA, Cavalcante IL, Araújo NS foram responsáveis pela coleta de dados e redação do artigo científico. Soares ACN foi responsável pela revisão da literatura. Serrão CCA foi responsável pela análise dos resultados. Almeida PSM, Nunes EFC foram responsáveis pela revisão do artigo.

\section{Competing interests}

No financial, legal or political competing interests with third parties (government, commercial, private foundation, etc.) were disclosed for any aspect of the submitted work (including but not limited to grants, data monitoring board, study design, manuscript preparation, statistical analysis, etc.).

\section{Referências}

1. Eickmeyer SM. Anatomy and Physiology of the Pelvic Floor. Phys Med Rehabil Clin N Am. 2017;28(3):455-60. https://doi. org/10.1016/j.pmr.2017.03.003

2. Hallock JL, Handa VL. The Epidemiology of Pelvic Floor Disorders and Childbirth: An Update. Obstet Gynecol Clin North Am. 2016;43(1):1-13. https://doi.org/10.1016/j.ogc.2015.10.008

3. Kovacs GT, Heath P, Heather C. First Australian trial of the birthtraining device Epi-No: a highly significantly increased chance of an intact perineum. Aust N ZJ Obstet Gynaecol 2004;44(4):347-8. https://doi.org/10.1111/j.1479-828x.2004.00265.x

4. Silva LM, Oliveira SMJV, Silva FMB, Alvarenga MB. Uso da bola suíça no trabalho de parto. Acta Paul Enferm. 2011;24(5):656-62. https://doi.org/10.1590/S0103-21002011000500010

5. Silva RB, Rios MJBL, Araújo JML, Sousa MD, Lago RBM, Barbosa IS. Atuação do fisioterapeuta no período gestacional: uma revisão integrativa de literatura. ReonFacema [Internet]. 2018;4(4):13308. Disponível em: https://www.facema.edu.br/ojs/index.php/ $\underline{\text { ReOnFacema/article/view/310 }}$

6. Nunes S, Varges OMC. A crioterapia como estratégia para alívio da dor no trabalho de parto: um estudo exploratório. $\mathrm{R}$ Enferm UERJ [Internet]. 2007;15(3):337-42. Disponível em: http:// www.luzimarteixeira.com.br/wp-content/uploads/2015/07/ CRIOTERAPIA-E-ALIVIO-DE-DOR.pdf

7. Pereira A, Oliveira AMB. Eficácia do EpiNo® na diminuição da episiotomia e risco de lesão perineal pós parto: revisão. Femina [Internet]. 2015;43(6). Disponível em: http://files.bvs.br/ upload/S/0100-7254/2015/v43n6/a5324.pdf 
8. Tamanini JTN, Dambros M, D'Ancona CAL, Palma PCR, Netto Júnior NR. Validação para o português do International Consultation on Incontinence Questionnaire Short Form (ICIQSF). Rev saude publica. 2004;38(3):438444. Disponível em: https://doi. org/10.1590/S0034-89102004000300015

9. Magno LDP, Pereira FAJ, Nunes EFC. Avaliação quantitativa da função sexual feminina correlacionada com a contração dos músculos do assoalho pélvico. Rev Pan-Amaz Saúde. 2011;2(4):3946. http://dx.doi.org/10.5123/S2176-62232011000400006

10. Martins L, Sousa ALL, Salgado CM. Prevalência da incontinência urinária em gestantes e puérperas em maternidades públicas. Fisioterapia Brasil [Internet]. 2017;11(5):334-9. Disponível em: https://portalatlanticaeditora. com.br/index.php/fisioterapiabrasil/article/view/1418/2565

11. Santini ACM, Santos ES, Vianna LS, Bernardes JM, Dias A. Prevalência e fatores associados à ocorrência de incontinência urinária na gestação. Rev Bras Saúde Mater Infant. 2019;19(4):967-74. https://doi.org/10.1590/1806$\underline{93042019000400013}$

12. Oliveira C, Seleme M, Cansi PF, Consentino RFDC, Kumakura FY, Moreira GA, et al. Incontinência urinária na gravidez e sua relação com as variáveis sociodemográficas e qualidade de vida. Rev Assoc Med Bras. 2013;59(5):460-6. https://doi.org/10.1016/j. ramb.2013.08.002

13. Bernardes NO. Incontinência urinária feminina e fatores de risco. Fisioterapia Brasil. 2018;7(4):301-6. https://doi. org/10.33233/fb.v7i4.1921

14. Spellacy CE. Urinary incontinence in pregnancy and the puerperium. J Obst Gynecol Neonatal Nurs. 2008;30(6):634-41. https://doi.org/10.1111/j.1552-6909.2001.tb00010.x

15. Valeton CT, Amaral VF. Evaluation of urinary incontinence in pregnancy and postpartum in Curitiba Mothers Program: a prospective study. Int Urogynecol J. 2011;22(7):813-8. https://doi. org/10.1007/s00192-011-1365-8

16. Lopes DBM, Praça NS. Incontinência urinária autorreferida no pós-parto. Texto contexto-enferm. 2010;19(4):667-74. https://doi. org/10.1590/S0104-07072010000400009

17. Faisal-Cury A, Menezes PR, Quayle J, Matijasevich A, Diniz SG. The relationship between mode of delivery and sexual health outcomes after childbirth. J Sex Med. 2015;12(5):1212-20. https:// doi.org/10.1111/jsm.12883

18. Silva TB, Bulhões TRB, Cirqueira RP, Ferreira JB. Análise da função sexual e imagem genital em primíparas e multíparas pósparto vaginal. Id on Line Ver Mult Psic [Internet]. 2017;11(39):97111. Disponível em: https://idonline.emnuvens.com.br/id/article/ view/982/1403
19. Pereira TRC, Dottori EH, Mendonça FMAF, Beleza ACS. Avaliação da função sexual feminina no puerpério remoto: um estudo transversal. Rev Bras Saúde Mater Infant. 2018;18(2):28994. https://doi.org/10.1590/1806-93042018000200003

20. Holanda JBL, Abuchaim JSV, Coca KP, Abrão ACFV. Disfunção sexual e fatores associados relatados no período pós-parto. Acta Paul Enferm. 2014;27(6):573-8. https://doi.org/10.1590/19820194201400093

21. O'Malley D, Higgins A, Begley C, Daly D, Smith V. Prevalence of and risk factors associated with sexual health issues in primiparous women at 6 and 12 months postpartum; a longitudinal prospective cohort study (the MAMMI study). BMC Pregnancy Childbirth. 2018;18(1):196. https://doi.org/10.1186/ s12884-018-1838-6

22. Marques J, Botelho S, Pereira LC, Lanza AH, Amorim CF, Palma $P$, et al. Pelvic floor muscle training program increases muscular contractility during first pregnancy and postpartum: electromyographic study. Neurourol Urodyn. 2013;32(7):9981003. https://doi.org/10.1002/nau.22346

23. Shek KL, Chantarasorn V, Langer S, Phipps H, Dietz HP. Does the Epi-No ${ }^{\circledR}$ Birth Trainer reduce levator trauma? A randomised controlled trial. Int Urogynecol J. 2011;22(12):1521-8. https://doi. org/10.1007/s00192-011-1517-x

24. Hillebrenner J, Wagenpfeil S, Schuchardt R, Schel - ling M, Scheneider KTM. First clinical experiences with the new birth trainer Epino $®$ in Primiparous women. Z Geburtsh Neonatol [Internet]. 2001;205(1):12-19. Disponível em: https://epino.nl/PDF/ first_clinical1_2.pdf

25. Kok J, Tan KH, Koh S, Cheng PS, Lim WY, Yew ML, et al. Antenatal use of a novel va - ginal birth training devide by term primiparous women in Singapore. Singapore Med J. 2004;45(7):31823. Citado em: PMID: 15221047.

26. Geranmayeh M, Rezaei Habibabadi Z, Fallahkish B, Farahani MA, Khakbazan Z, Mehran A. Reducing perineal trauma through perineal massage with vaseline in second stage of labor. Arch Gynecol Obstet. 2012;285(1):77-81. https://doi.org/10.1007/ s00404-011-1919-5

27. Cardozo CIA, Cunha FMAM. Avaliação do impacto de um protocolo fisioterapêutico na diminuição do quadro álgico durante a primeira fase do trabalho de parto vaginal. Fisioterapia Brasil [Internet]. 2019;20(2):222-9. Disponível em: https:// portalatlanticaeditora.com.br/index.php/fisioterapiabrasil/article/ view/2233 